\title{
Methodological Issues in Preclinical Mouse Efficacy Studies of Adenomyosis
}

\author{
Sun-Wei Guo
}

Published online: 30 June 2012

(C) Springer Science+Business Media, LLC 2012

\begin{abstract}
Adenomyosis is a benign, non-neoplastic gynecologic disorder with a poorly understood pathogenesis. Its treatment has been a challenge, with hysterectomy being considered the definitive therapy for severe adenomyosis. More efficacious drugs with better side-effect and cost profiles are sorely needed. Unfortunately, there is a dearth of registered clinical trials on adenomyosis, and the registered trials are not testing new compounds. This appears to suggest a loss in translating discoveries made in preclinical studies to clinical practice. This article presents a systematic review of all published mouse efficacy studies of adenomyosis aimed at the evaluation of potential therapeutic compounds, focusing on methodological quality and describing the limitations of the published studies. The complex issues involved in translating preclinical studies to clinical practice will be summarized. Lastly, some methodological issues in mouse efficacy studies of adenomyosis will be highlighted, with the aim to improve such studies in the future.
\end{abstract}

Keywords Adenomyosis · Animal studies · Drug development $\cdot$ Efficacy $\cdot$ Methodological quality $\cdot$ Mouse . Outcome measures

\section{Introduction}

Adenomyosis is a benign, non-neoplastic gynecologic disorder characterized by the ectopic proliferation of endometrial

\section{S.-W. Guo $(\bowtie)$}

Shanghai Obstetrics and Gynecology Hospital, Fudan University, 419 Fangxie Road,

Shanghai 200011, China

e-mail: hoxa10@gmail.com tissue into the myometrium with smooth muscle hypertrophy. A common disorder with a prevalence in hysterectomy specimens ranging from $14 \%$ to $66 \%$ [1], its common presenting symptoms include abnormal uterine bleeding, dysmenorrhea, and subfertility [2], although a sizeable portion of patients are asymptomatic [3]. Dysmenorrhea is the most debilitating symptom [4]. Treatment of adenomyosis has been a challenge, with hysterectomy being considered the definitive therapy for severe adenomyosis [5]. Uterine artery embolization (UAE) also has shown promise, but a recent review noted that the recurrence risk after UAE is high [6].

Although adenomyosis is hormone-sensitive, progestogenic agents are not very effective, and gonadotrophinreleasing hormone $(\mathrm{GnRH})$ agonists induce suppression of adenomyosis, yet their use is restricted by short duration [7]. In addition, the symptoms quickly reappear after discontinuation of GnRH agonist therapy [8]. While the insertion of a levonorgestrel-releasing intrauterine system (LNG-IUS) has been reported to result in a reduction in adenomyosisassociated pain and abnormal bleeding [9], side effects such as spotting are reported in one third of the women, with many women experiencing oligomenorrhea [9]. Consequently, more efficacious drugs with better side-effect and cost profiles are sorely needed.

Despite this unmet medical need, a search of clinical trial registries suggests few new agents are being investigated. Compared with 1,679 trials on ovarian cancer, 146 on uterine fibroids, and 132 on endometriosis, only 15 trials on adenomyosis were registered (www.ClinicalTrials.gov, accessed on June 5, 2012). Among the 15 trials, 1 was not yet recruiting, 1 was active but not recruiting, 3 were completed, 4 were recruiting, and 6 had an "unknown" status, meaning that "the trial status has not been verified in more than 2 years." Of the 15 trials, only 4 trials evaluated 
nonsurgical treatment: one on progestin, one on acupuncture, one on LUS-IUD, and one on letrozole, an aromatase inhibitor. There are no novel compounds currently under trial evaluation. The completed trial on letrozole indicated that its efficacy, evaluated 12 weeks after treatment, was comparable with the GnRH agonist [10]. Thus, the research and development (R\&D) for medical treatment of adenomyosis seem to be limited.

One indispensable component of preclinical research in drug R\&D is animal efficacy studies. This review will focus on the methodology of mouse efficacy studies through a systematic review of all published mouse efficacy studies of adenomyosis evaluating potential therapeutic compounds and will describe the limitations in these studies. With the exception of danazol and danazol-containing intrauterine devices (IUDs) that were tested clinically before mouse studies, no compound evaluated preclinically has been tested clinically or in clinical trials, at least not within the public domain. The completed trial on letrozole was conducted without an animal study demonstrating its therapeutic potential. Many complex issues are involved in translating preclinical studies to clinical practice. Some methodological aspects of preclinical animal studies of adenomyosis will be reviewed, with the aim to improve mouse efficacy studies of adenomyosis.

\section{Methods}

A systematic and comprehensive search of PubMed was performed for all studies published up to June 5, 2012, using the following search terms: "adenomyosis" or "endometriosis" in combination with "mouse," "mice," "rat," "rodent," or "animal model." Included studies used rodent models of adenomyosis, not endometriosis and evaluated the therapeutic potential of a compound or compounds. The search was limited to publications written in English. Thus, articles on endometriosis, but not adenomyosis per se, were excluded. The focus on mouse studies is of necessity because no other animal models of adenomyosis have been used for efficacy studies.

The search resulted in 14 studies of mouse models of adenomyosis [11-20, 21 , 22, 23•, 24]. Of the 14 studies, four were excluded because one study started the intervention from the day of birth [24], one reported an intervention that was not effective [13], one actually focused on mammary tumors and mentioned adenomyosis as a secondary end point [15], one that tested the same compound that was evaluated previously [16], and one study to test feasibility, but not efficacy, of an intervention [20]. The characteristics of the remaining ten studies, along with the major treatment outcome measures in the control group and the group receiving the highest dose, representing the best treatment effect, are listed in Table 1.

\section{Methods of Inducing Adenomyosis in Mouse Studies}

Greaves and White [26] recently reviewed animal models of adenomyosis, including mouse models. Briefly, adenomyosis can be induced in mice through various forms of hormonal imbalance, such as hyperprolactinemia [27, 28], prolonged treatment with or prenatal exposure to estrogenic compounds [27, 29], and prolonged treatment with progesterone $[30,31]$. For efficacy studies, however, none of these induction methods have been used. One reason is the long induction period. The other reason is their nonspecific effect, such that in addition to inducing adenomyosis, other pathological changes in the reproductive tract are also induced. For example, prenatal exposure to diethylstilbestrol also results in cervix enlargement with extensive mucoid alterations within the muscularis [27].

For efficacy studies, some published studies used the SHN mouse strain, which has spontaneous adenomyosis [32]. Others have used one of two adenomyosis induction methods, ectopic grafting of pituitary glands (EGPG) or neonatal feeding with tamoxifen (NFT). In EGPG, adenomyosis is induced by intrauterine implantation of isographs of anterior pituitary glands [33-35]. This induction method has the advantage of a high success rate, reported to be greater than $90 \% 50$ days after induction [16], and the ultrastructural changes in myometrium from mice with induced adenomyosis have been documented [36]. The limitations of this approach include that the induction procedure requires a certain level of surgical skill such that surgical changes might be a confounding effect in efficacy studies. A second limitation is that the success rate is strain dependent, with high rates in SHN and SLN mouse strains but lower rates in other strains $[34,35]$. A third limitation is the long induction period of 4 months in an strain of ICR mice to reach a success rate of $95 \%$ [21•] and a 6-month induction period in $\mathrm{C} 3 \mathrm{H}$ and $\mathrm{F} 1$ hybrids with a variable success rate [35]. Lastly, the induction method appears to be sensitive to treatment with dimethyl sulfoxide (DMSO), often used as a vehicle for many compounds. In Dr. Mori's lab, the success rate of adenomyosis induction after EGPG followed by DMSO treatment was reduced to $80 \%$ in one study [18] and $40 \%$ in another [19]. The anti-inflammatory effect of DMSO may be related to its use to treat intractable interstitial cystitis, radiation cystitis, chronic prostatitis, and chronic female trigonitis [37]. DMSO also is used most as a topical analgesic [38]. Thus, if the induction is truly sensitive to DMSO, an underestimation of the efficacy of studied compounds may occur when DMSO is used as a solvent.

Neonatal feeding with tamoxifen was first reported by Parrott et al. $[39,40]$ in CD-1 mouse. A previous study of neonatal exposure to tamoxifen, delivered through subcutaneous injection, in the CD-1 mouse resulted in atypical hyperplasia in the endometrium, uterine adenocarcinoma, 


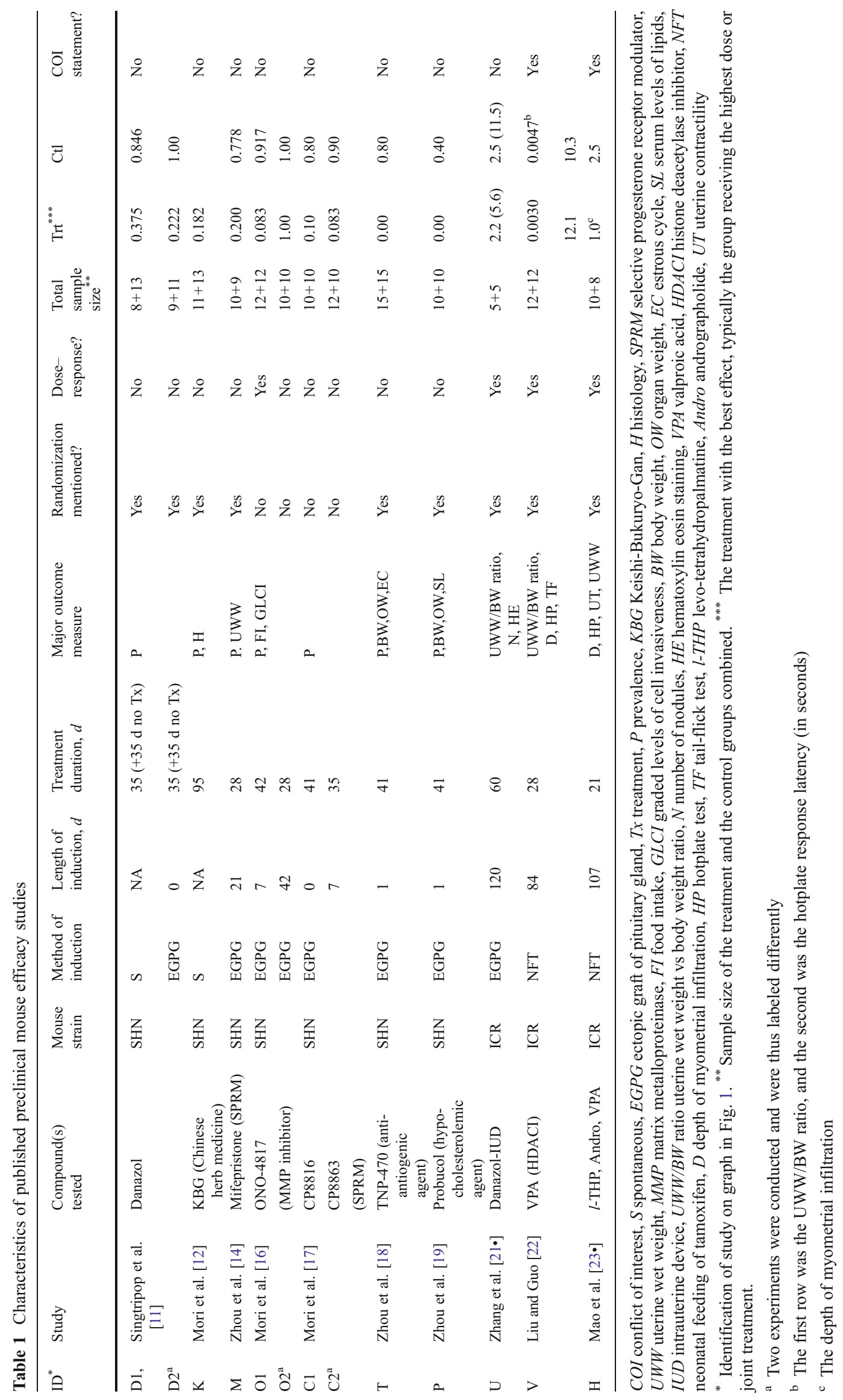


and uterine hypoplasia with focal areas of basal cell hyperplasia in the endometrium, but not adenomyosis [41]. Because the ICR mouse is genetically related with the CD-1 mouse, NFT with the ICR mouse yielded identical results in inducing adenomyosis [22]. Compared with the EGPG method, NFT is much simpler, requiring no surgical skill. In addition, it also has a high success rate in induction: 3 months after the induction procedure, all mice have adenomyosis $[22,40]$. In fact, by day 42 or 37 days after the NFT, the success rate is $100 \%$ [42]. Like the EGPG method, the NFT also has the limitation that the success of the induction is highly strain dependent [43].

\section{Features of Published Mouse Efficacy Studies}

From Table 1, several notable features can be seen. First, the earlier (up to 2004) mouse efficacy studies were conducted exclusively in Japan, specifically, in Dr. Mori's lab in the University of Tokyo, which first reported that EGPG resulted in a high rate of development of adenomyosis [44]. In SHN mouse strain, which has a high incidence of mammary tumors, an unusually high incidence of spontaneous adenomyosis also was reported [45]. In this model, an increase in plasma levels of prolactin is noted [28].

Diverse compounds have been evaluated for their therapeutic potential. These included danazol, a Chinese herb concoction, a matrix metalloproteinase (MMP) inhibitor (ONO-4817), several selective progesterone receptor modulators (SPRMs [mifepristone, CP-8816, and CP-8863]), a danazol-containing IUD, a histone deacetylase inhibitor (valproic acid [VPA]), an anti-angiogenic agent (TNP470); a hypocholesterolemic agent (probucol), an analgesic (levo-tetrahydropalmatine [1-THP]), and a nuclear factor (NF)- $\mathrm{KB}$ inhibitor (andrographolide). Of these, only danazol [46], VPA [47, 48], and andrographolide [49], have been evaluated in in vitro or clinical studies for endometriosis or adenomyosis. Despite promising results, only danazol has been tested in clinical trials or is in use clinically today. However, adenomyosis usually fails to respond to oral danazol therapy [50].

Earlier studies were focused on biological plausibility. For example, because adenomyosis involves cell migration or invasion, which is associated with the degradation and reconstruction of the extracellular matrix in which MMPs are involved, MMP inhibitors were studied. In contrast, more recent, studies have been designed based on previous molecular studies. For example, because adenomyosis may be an epigenetic disease [51], it may be treatable by histone deacetylase inhibitors similar to endometriosis [52]. Consequently, VPA recently has been tested in animal models of adenomyosis [22]. Interestingly, clinical studies of oral administration of VPA in treating symptomatic adenomyosis have yielded promising results $[48,53]$. Andrographolide is an NF-KB inhibitor that causes covalent modification of reduced cysteine 62 of the p50 subunit of NF-kB [54] and may also be therapeutic given the constitutive NF- $\mathrm{KB}$ activation in adenomyosis [55]. Tetrahydropalmatine (THP) is an alkaloid compound and a main active ingredient of Corydalis yanhusuo W. T. Wang [56], a well-known analgesic in traditional Chinese medicine (TCM) often used as a constituent herb for treating endometriosis [57]. Its levoenantiomer ( $l$-THP) is an analgesic with remarkable sedative tranquilizing but nonaddictive effects [58]. Listed in the Chinese Pharmacopoeia since 1977, l-THP has been used as a sedative or analgesic for chronic pains in China. The danazol-containing IUD and vaginal ring were tested clinically for adenomyosis [50, 59, 60] and endometriosis [61]. On a molecular level, danazol treatment results in reduced expression of estrogen receptor and bcl-2 in adenomyotic endometrium with concomitant increased apoptosis [62].

Over time, the clinical outcome in animal models has evolved from using only the prevalence of adenomyosis to including details such as the ratio of uterine wet weight versus total body weight [21•], number of nodules [21•], or depth of myometrial infiltration of endometrial cells [22, 23•] as outcome measures. Additionally, two recent studies used latency response to noxious thermal stimuli as outcome measures $[22,23 \bullet]$. Using adenomyosis prevalence as an outcome measure is useful in determining suppression or slowed development of adenomyosis. However, because women with endometriosis and a prolong history of dysmenorrhea often have adenomyosis [63], ideal compounds will reverse adenomyosis and treat associated symptoms.

\section{Analysis of Earlier Mouse Studies}

As seen in Table 1, the seven mouse efficacy studies published up to 2004 were conducted by one research group using the SHN mouse strain and evaluated different compounds. The induction methods and major outcomes were nearly identical. The three later studies from $2008(n=1)$ and $2011(n=2)$, used different strains of mice or different induction methods. The outcome measures also differed from earlier studies.

An analysis was conducted to determine whether the magnitude of treatment effect, defined as the difference in prevalence of adenomyosis between the treatment and control groups, was associated with features of the experiment. Comparison of the distributions among two or more groups of continuous variables was made using the Wilcoxon and Kruskal-Wallis tests, respectively. Pearson's or Spearman's rank correlation coefficient was used when evaluating correlations between two variables when both variables were continuous or when at least one variable was ordinal. To see 
whether the age of mice used in the experiment, the length of adenomyosis-induction period, duration of treatment, and total sample size were responsible for the reduction in prevalence of adenomyosis between treatment and control groups, a multiple linear regression model was used. $P$ values of less than 0.05 were considered statistically significant. All computations were made with R statistics software system version 2.15.0 [25].

As illustrated in Fig. 1, the treatment effect was inversely correlated with the length of induction period (ie, the time between performing the pituitary graft and the start of treatment $[r=0.79, P=0.02$; Fig.1A $]$ ). In other words, better efficacy was reported from experiments using a shorter induction period, or earlier intervention.

Improved efficacy also seemed to be associated with longer duration of treatment, although the correlation did not reach statistical significance $(r=-0.38, P=0.38$ [Fig. 1B]). Greater reduction in prevalence tended to be reported from experiments that did not mention randomization in allocating animals, but no statistical significance was reached ( $P=0.61$ [Fig. $1 \mathrm{C}]$ ).

A multiple linear regression analysis using the difference in prevalence of adenomyosis between the treatment and control groups as the independent variable and the age of mice used in the experiment, year of publication, length of induction period, duration of treatment, total sample size, and the mention of randomization as dependent variables revealed that the length of induction period was the only covariate that was significantly associated with the reduction in prevalence $\left(P=0.035, R^{2}=0.78\right)$ indicating that greater efficacy is associated with earlier intervention.

\section{Methodological Qualities of Published Mouse Efficacy Studies}

The recently proposed Animals in Research: Reporting In Vivo Experiments (ARRIVE) guidelines [64・•] comprise seven core categories that indicate methodological qualities of animal efficacy studies. These categories for the ten reviewed studies are summarized in Table 2. In considering these core elements, none of the reviewed studies provide any justification for number of mice studied or how the sample size was determined. Eight of ten studies mentioned that the animal allocation was carried out randomly, but none described how it was done. None of the reviewed studies reported allocation concealment. None of the ten studies explicitly stated that the outcome measures were evaluated blinded to treatment allocation. Of the ten studies, four used two or more dosages to establish the minimally effective or maximally tolerated doses. Because the two major complaints from women with adenomyosis are pain and heavy menstrual bleeding, one needs to evaluate not
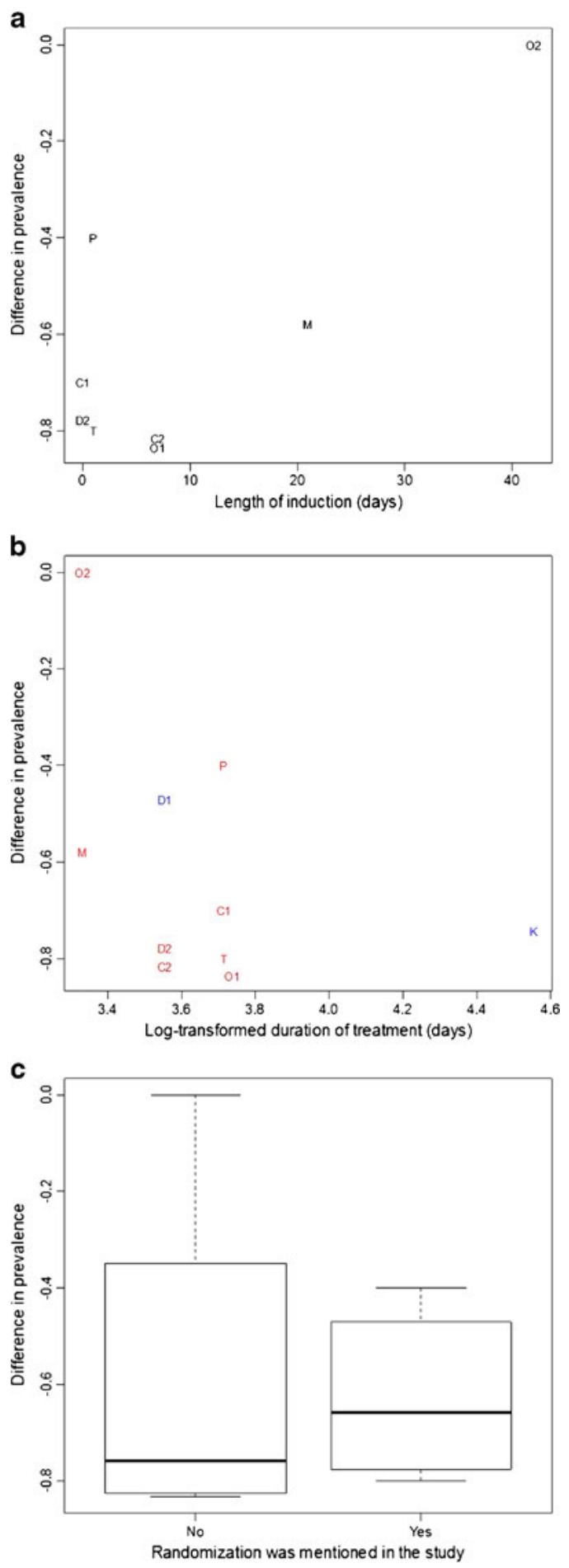

Fig. 1 a Scatter plot of the difference in incidence of adenomyosis between treatment and control groups versus length of induction. The alphabets were identifications of experiments listed in Table 1. b Scatter plot of the difference in prevalence of adenomyosis between treatment and control groups versus duration of treatment (in days). The alphabets were identifications of experiments listed in Table 1. The experiments with blue alphabets were done with spontaneous adenomyosis in SHN mice, while those with red were performed with ectopic graft of pituitary glands. c Box plot of the difference in incidence of adenomyosis between treatment and control groups by the statement of randomization in allocating mice in experiment 
only the histological outcome of an intervention (ie, number of nodules, depth of myometrial infiltration, with or without proliferation index measurement or immunostaining analysis) but also functional outcome (ie, severity of pain or fertility). While eight of ten studies evaluated at least two diseaserelated outcomes, only two of those eight studies evaluated functional outcomes (ie, pain behavior). Of the ten studies, only two recently published studies addressed possible conflict of interest (COI). The lack of a statement regarding COI in the earlier studies may reflect their publication at an earlier time when disclosure regarding COI was not required.

The importance of the methodological quality in animal studies has been appreciated in other clinical settings, such as stroke, after some failed clinical trials [66-68]. Rigorous, robust, and detailed preclinical evaluation has been recognized as a critical step in the development of effective novel therapies [66]. The less than desirable methodological quality of mouse efficacy studies of adenomyosis illustrated in Table 2 could have contributes to the lack of progress in developing novel treatments for adenomyosis. The scarcity of registered clinical trials on adenomyosis may be attributable, at least in part, to various deficiencies in mouse efficacy studies.

The tendency to alter animal numbers based on scientifically irrelevant issues such as availability or cost has been noted before [69]. Inadequate sample sizes would result in underpowered studies, bringing into question the statistical validity of study conclusions. For continuous outcome measures, the rule of thumb for calculating the desired sample size $n$ to achieve the significance level of 0.05 and $80 \%$ power in the treatment and the control group is $n-1=16 \mathrm{~s}^{2} / \mathrm{d}^{2}$, where $s$ is the standard deviation, and $d$ is the difference to be detected [70]. In other words, $n-1$ is proportional to the squared standard deviation, but inversely proportional to the squared difference to be detected. Without knowing $s$ and/or $d$, sample size determination is impossible. Ideally, pilot experiments would aid in determining $s$ and/or $d$. In some cases, this approach would result in doing the entire experiment. Therefore, this quandary may explain why in many mouse efficacy

Table 2 Summary of core quality features of ten published mouse efficacy studies of adenomyosis

\begin{tabular}{ll}
\hline Category & Fulfilled, \% \\
\hline Sample size justification & 0 \\
Randomization & 80 \\
Allocation concealment & 0 \\
Blinding & 0 \\
Does-response relationship & 40 \\
Assessment of at least one functional outcome & 20 \\
Statement regarding possible conflicting interest & 20 \\
\hline
\end{tabular}

studies, sample size justification is not done or is carried out post hoc.

Randomization and allocation concealment can be done in mouse efficacy studies. Blinding also can be done, but when there is a shortage of hands, as in experiments for a student's $\mathrm{PhD}$ dissertation, extra care should be taken to meet this requirement. Declaration of potential COI is now mandatory for publication in many biomedical journals. When a preclinical study is sponsored or even conducted by a drug company testing a compound developed by the company itself, there is obviously vested interest in the results of the tested compound and, as such, potential COI should disclosed and declared.

\section{Conclusions}

Preclinical mouse efficacy studies of adenomyosis apparently have deficiencies in methodology. Critical evaluation of published data before embarking on animal studies, the use of mouse models that adequately mimic human adenomyosis, the use of at least one functional outcome, and higher methodological quality could greatly increase the chance of a successful translation of mouse experiments into clinical success. Fortunately, the importance of methodological quality of animal efficacy studies is now well recognized, and, as a result, the ARRIVE guidelines for experimental design, execution, and reporting have been established [65•]. The use of these guidelines in future mouse efficacy studies of adenomyosis would strengthen preclinical research.

Adenomyosis is a difficult disease to manage [65], especially by nonsurgical means. Developing novel, more efficacious therapeutics with better safety and cost profiles is a pressing but unmet medical need. On www.ClinicalTrials. gov, the number of registered clinical trials studying adenomyosis is currently only 15 , about one tenth of the number of trials on endometriosis; none are testing novel drugs. The amount of research on adenomyosis indicated by PubMed using the phrase "adenomyosis not endometriosis" yielded 389 publications, as compared with 18,593 when the search used the word "endometriosis" alone (PubMed, accessed on May 14, 2012). Insufficient breadth of research regarding adenomyosis may hamper compiling information relevant to drug development.

Besides the methodological quality as discussed above, proceeding from preclinical studies to clinical trials requires that efficacy is reproduced in two laboratories, one of which is independent of any sponsoring company [66]. In addition, the use of second larger species such as cats, dogs, or primates would be highly valuable, but, unfortunately, no such animal model is available now. Only when the preclinical mouse efficacy study is carried out with methodological 
rigor and careful consideration will there be a better chance to develop a successful drug to treat adenomyosis.

Acknowledgment This research was supported in part by grants 09PJD015 and 10410700200 from the Shanghai Science and Technology Commission, and support from Shanghai Key Laboratory of Female Reproductive Endocrine-Related Diseases and from the Key Specialty Project of the Ministry of Health, People's Republic of China. The author thanks Dr. Pamela Stratton for her constructive comments on an earlier version of this manuscript.

Disclosures No potential conflicts of interest relevant to this article were reported.

\section{References}

Papers of particular interest, published recently, have been highlighted as:

- Of importance

•. Of major importance

1. Vercellini P, Vigano P, Somigliana E, Daguati R, Abbiati A, Fedele L. Adenomyosis: epidemiological factors. Best Pract Res Clin Obstet Gynaecol. 2006;20:465-77.

2. Farquhar C, Brosens I. Medical and surgical management of adenomyosis. Best Pract Res Clin Obstet Gynaecol. 2006.

3. Benson RC, Sneeden VD. Adenomyosis: a reappraisal of symptomatology. Am J Obstet Gynecol. 1958;76:1044-57. discussion 1057-61.

4. Matalliotakis IM, Kourtis AI, Panidis DK. Adenomyosis. Obstet Gynecol Clin North Am. 2003;30:63-82. viii.

5. Wood C. Surgical and medical treatment of adenomyosis. Hum Reprod Update. 1998;4:323-36.

6. Englander MJ. Uterine artery embolization for the treatment of adenomyosis. Semin Intervent Radiol. 2008;25:387-93.

7. Bergeron C, Amant F, Ferenczy A. Pathology and physiopathology of adenomyosis. Best Pract Res Clin Obstet Gynaecol. 2006;20:511-21.

8. Grow DR, Filer RB. Treatment of adenomyosis with long-term GnRH analogues: a case report. Obstet Gynecol. 1991;78:538-9.

9. Bragheto AM, Caserta N, Bahamondes L, Petta CA. Effectiveness of the levonorgestrel-releasing intrauterine system in the treatment of adenomyosis diagnosed and monitored by magnetic resonance imaging. Contraception. 2007;76:195-9.

10. Badawy AM, Elnashar AM, Mosbah AA. Aromatase inhibitors or gonadotropin-releasing hormone agonists for the management of uterine adenomyosis: a randomized controlled trial. Acta Obstet Gynecol Scand. 2012.

11. Singtripop T, Mori T, Sakamoto S, Sassa S, Park MK, Kawashima S. Suppression of the development of uterine adenomyosis by danazol treatment in mice. Life Sci. 1992;51:1119-25.

12. Mori T, Sakamoto S, Singtripop T, Park MK, Kato T, Kawashima $\mathrm{S}$, Nagasawa H. Suppression of spontaneous development of uterine adenomyosis by a Chinese herbal medicine, keishi-bukuryogan, in mice. Planta Med. 1993;59:308-11.

13. Nagasawa H, Hirayama T, Inatomi H. Effects of simultaneous treatment with hydroxyapatite and coffee cherry, the residue left after the removal of coffee beans, on spontaneous mammary tumourigenesis and related parameters in SHN mice. In Vivo. 1999;13:385-8.

14. Zhou YF, Matsuda M, Mori T, Sakamoto S, Mitamura T. Effects of mifepristone (RU486) treatment on the development of uterine adenomyosis induced by pituitary grafting in mice. Life Sci. 2000;67:2713-20.
15. Mori T, Nakahashi K, Kyokuwa M, Yamasaki S, Nagasawa H. A matrix metalloproteinase inhibitor, ONO-4817, retards the development of mammary tumor and the progression of uterine adenomyosis in mice. Anticancer Res. 2002;22:3985-8.

16. Mori T, Yamasaki S, Masui F, Matsuda M, Sasabe H, Zhou YF. Suppression of the development of experimentally induced uterine adenomyosis by a novel matrix metalloproteinase inhibitor, ONO4817, in mice. Exp Biol Med (Maywood). 2001;226:429-33.

17. Mori T, Kurata Y, Tabata Y, Niho N, Matsuda M, Zhou YF. Priming effects of novel nonsteroidal progesterone receptor modulators CP8816 and CP8863 on the development of adenomyosis in the mouse uterus. Life Sci. 2002;71:527-35.

18. Zhou YF, Mori T, Kudo H, Asakai R, Sassa S, Sakamoto S. Effects of angiogenesis inhibitor TNP-470 on the development of uterine adenomyosis in mice. Fertil Steril. 2003;80 Suppl 2:788-94.

19. Zhou YF, Mori T, Nagasawa H, Nakayama T, Kubota T, Sakamoto S. Probucol, a hypocholesterolemic agent, prevents the development of uterine adenomyosis induced by pituitary grafting in mice. Anticancer Res. 2004;24:2209-12.

20. Suzuki-Kakisaka H, Murakami T, Hirano T, Terada Y, Yaegashi N, Okamura K. Selective accumulation of PpIX and photodynamic effect after aminolevulinic acid treatment of human adenomyosis xenografts in nude mice. Fertil Steril. 2008;90:1523-7.

21. - Zhang X, Yuan H, Deng L, Hu F, Ma J, Lin J. Evaluation of the efficacy of a danazol-loaded intrauterine contraceptive device on adenomyosis in an ICR mouse model. Hum Reprod. 2008;23:2024-30. This is the first report of using ICR mouse, instead of SHN mouse, to induce adenomyosis via ectopic grafting of pituitary glands. The evaluation of multiple dosage forms and of different delivery routes are features of this study.

22. Liu X, Guo SW. Valproic acid alleviates generalized hyperalgesia in mice with induced adenomyosis. J Obstet Gynaecol Res. 2011;37:696-708.

23. - Mao X, Wang Y, Carter AV, Zhen X, Guo SW. The retardation of myometrial infiltration, reduction of uterine contractility, and alleviation of generalized hyperalgesia in mice with induced adenomyosis by levo-tetrahydropalmatine (1-THP) and andrographolide. Reprod Sci. 2011;18:1025-37. This is the first report on the use of ICR mouse, instead of CD-1 mouse, for mouse model of adenomyosis. It makes use of this model to evaluate the efficacy of two different compounds and of their joint treatment, a sort of polypill. This study also employs hotplate test as a proxy measure of adenomyosis-induced pain. In addition, it measures the uterine contractility and correlates with the change in pain behavior.

24. Nagasawa H, Noguchi Y, Mori T, Niki K, Namiki H. Suppression of normal and preneoplastic mammary growth and uterine adenomyosis with reduced growth hormone level in SHN mice given monosodium glutamate neonatally. Eur J Cancer Clin Oncol. 1985;21:1547-51.

25. Team RDC. R: A language and environment for statistical computing. R Foundation for Statistical Computing, Vienna, Austria; 2009.

26. Greaves P, White IN. Experimental adenomyosis. Best Pract Res Clin Obstet Gynaecol. 2006;20:503-10.

27. Huseby RA, Thurlow S. Effects of prenatal exposure of mice to "low-dose" diethylstilbestrol and the development of adenomyosis associated with evidence of hyperprolactinemia. Am J Obstet Gynecol. 1982;144:939-49.

28. Mori T, Singtripop T, Kawashima S. Animal model of uterine adenomyosis: is prolactin a potent inducer of adenomyosis in mice? Am J Obstet Gynecol. 1991;165:232-4.

29. Guttner J. Adenomyosis in mice. Z Versuchstierkd. 1980;22:249-51.

30. Lipschutz A, Iglesias R, Panasevich VI, Salinas S. Pathological changes induced in the uterus of mice with the prolonged administration of progesterone and 19-nor-contraceptives. Br J Cancer. 1967;21:160-5.

31. Ostrander PL, Mills KT, Bern HA. Long-term responses of the mouse uterus to neonatal diethylstilbestrol treatment and to later sex hormone exposure. J Natl Cancer Inst. 1985;74:121-35. 
32. Mori T, Nagasawa H. Alteration of the development of mammary hyperplastic alveolar nodules and uterine adenomyosis in SHN mice by different schedules of treatment with CB-154. Acta Endocrinol (Copenh). 1984;107:245-9.

33. Mori T, Nagasawa H. Mechanisms of development of prolactininduced adenomyosis in mice. Acta Anat (Basel). 1983;116:46-54.

34. Mori T, Nagasawa H, Nakajima Y. Strain-difference in the induction of adenomyosis by intrauterine pituitary grafting in mice. Lab Anim Sci. 1982;32:40-1.

35. Huseby RA, Soares MJ, Talamantes F. Ectopic pituitary grafts in mice: hormone levels, effects on fertility, and the development of adenomyosis uteri, prolactinomas, and mammary carcinomas. Endocrinology. 1985;116:1440-8.

36. Mori T, Ohta Y, Nagasawa H. Ultrastructural changes in uterine myometrium of mice with experimentally-induced adenomyosis. Experientia. 1984;40:1385-7.

37. Shirley SW, Stewart BH, Mirelman S. Dimethyl sulfoxide in treatment of inflammatory genitourinary disorders. Urology. 1978;11:215-20.

38. Swanson BN. Medical use of dimethyl sulfoxide (DMSO). Rev Clin Basic Pharm. 1985;5:1-33.

39. Green AR, Styles JA, Parrott EL, Gray D, Edwards RE, Smith AG, Gant TW, Greaves P, Al-Azzawi F, White IN. Neonatal tamoxifen treatment of mice leads to adenomyosis but not uterine cancer. Exp Toxicol Pathol. 2005;56:255-63.

40. Parrott E, Butterworth M, Green A, White IN, Greaves P. Adenomyosis-a result of disordered stromal differentiation. Am J Pathol. 2001;159:623-30.

41. Newbold RR, Jefferson WN, Padilla-Burgos E, Bullock BC. Uterine carcinoma in mice treated neonatally with tamoxifen. Carcinogenesis. 1997; 18:2293-8.

42. Mehasseb MK, Bell SC, Habiba MA. The effects of tamoxifen and estradiol on myometrial differentiation and organization during early uterine development in the CD1 mouse. Reproduction. 2009;138:341-50.

43. Mehasseb MK, Bell SC, Habiba MA. Neonatal administration of tamoxifen causes disruption of myometrial development but not adenomyosis in the C57/BL6J mouse. Reproduction. 2010;139:1067-75.

44. Mori T, Nagasawa H, Takahashi S. The induction of adenomyosis in mice by intrauterine pituitary isografts. Life Sci. 1981;29:1277-82.

45. Nagasawa H, Konishi R, Naito T, Ohmiya S, Mori T. Relationship between mammary tumourigenesis and uterine adenomyosis in four strains of mice. In Vivo. 1987;1:237-40.

46. Friedlander RL. The treatment of endometriosis with Danazol. J Reprod Med. 1973;10:197-9.

47. Wu Y, Guo SW. Histone deacetylase inhibitors trichostatin A and valproic acid induce cell cycle arrest and p21 expression in immortalized human endometrial stromal cells. Eur J Obstet Gynecol Reprod Biol. 2008;137:198-203.

48. Liu X, Guo SW. A pilot study on the off-label use of valproic acid to treat adenomyosis. Fertil Steril. 2008;89:246-50.

49. Zheng Y, Liu X, Guo SW. Therapeutic potential of andrographolide for treating endometriosis. Hum Reprod. 2012;27:1300-13.

50. Igarashi M. A new therapy for pelvic endometriosis and uterine adenomyosis: local effect of vaginal and intrauterine danazol application. Asia Oceania J Obstet Gynaecol. 1990;16:1-12.

51. Jichan N, Xishi L, Guo SW. Promoter hypermethylation of progesterone receptor isoform $\mathrm{B}$ (PR-B) in adenomyosis and its rectification by a histone deacetylase inhibitor and a demethylation agent. Reprod Sci. 2010;17:995-1005.
52. Guo SW. Epigenetics of endometriosis. Mol Hum Reprod. 2009;15:587-607.

53. Liu XS, Lei Y, Guo SW. Valproic acid as a therapy for adenomyosis: a comparative case series. Reprod Sci. 2010;17:904-12.

54. Xia YF, Ye BQ, Li YD, Wang JG, He XJ, Lin X, Yao X, Ma D, Slungaard A, Hebbel RP, Key NS, Geng JG. Andrographolide attenuates inflammation by inhibition of NF-kappa B activation through covalent modification of reduced cysteine 62 of p50. J Immunol. 2004;173:4207-17.

55. Nie J, Lu Y, Liu X, Guo SW. Immunoreactivity of progesterone receptor isoform $\mathrm{B}$, nuclear factor kappaB, and IkappaBalpha in adenomyosis. Fertil Steril. 2009;92:886-9.

56. Hu JY, Jin GZ. Effect of tetrahydropalmatine analogs on Fos expression induced by formalin-pain. Zhongguo Yao Li Xue Bao. 1999;20:193-200.

57. Han YF, Hou LH, Zhou YJ, Wu XK. A survey of TCM treatment for endometriosis. J Tradit Chin Med. 2009;29:64-70.

58. Chu H, Jin G, Friedman E, Zhen X. Recent development in studies of tetrahydroprotoberberines: mechanism in antinociception and drug addiction. Cell Mol Neurobiol. 2008;28:491-9.

59. Igarashi M, Abe Y, Fukuda M, Ando A, Miyasaka M, Yoshida M, Shawki OA. Novel conservative medical therapy for uterine adenomyosis with a danazol-loaded intrauterine device. Fertil Steril. 2000;74:412-3.

60. Tamaoka Y, Orikasa H, Sumi Y, Sakakura K, Kamei K, Nagatani M, Ezawa S. Direct effect of danazol on endometrial hyperplasia in adenomyotic women: treatment with danazol containing intrauterine device. Hum Cell. 2000;13:127-33.

61. Cobellis L, Razzi S, Fava A, Severi FM, Igarashi M, Petraglia F. A danazol-loaded intrauterine device decreases dysmenorrhea, pelvic pain, and dyspareunia associated with endometriosis. Fertil Steril. 2004;82:239-40

62. Ueki K, Kumagai K, Yamashita H, Li ZL, Ueki M, Otsuki Y. Expression of apoptosis-related proteins in adenomyotic uteri treated with danazol and GnRH agonists. Int J Gynecol Pathol. 2004:23:248-58.

63. Kissler S, Zangos S, Kohl J, Wiegratz I, Rody A, Gatje R, Vogl TJ, Kunz G, Leyendecker G, Kaufmann M. Duration of dysmenorrhoea and extent of adenomyosis visualised by magnetic resonance imaging. Eur J Obstet Gynecol Reprod Biol. 2008;137:204-9.

64. •• Kilkenny C, Browne WJ, Cuthill IC, Emerson M, Altman DG. Improving bioscience research reporting: the ARRIVE guidelines for reporting animal research. PLoS Biol. 2010;8:e1000412. This article spells out the ARRIVE guidelines.

65. Wood C. Adenomyosis: difficult to diagnose, and difficult to treat. Diagn Ther Endosc. 2001;7:89-95.

66. (STAIR), S. T. A. I. R. Recommendations for standards regarding preclinical neuroprotective and restorative drug development. Stroke. 1999;30:2752-8.

67. Dirnagl U. Bench to bedside: the quest for quality in experimental stroke research. J Cereb Blood Flow Metab. 2006;26:1465-78.

68. O'Collins VE, Macleod MR, Donnan GA, Horky LL, van der Worp BH, Howells DW. 1,026 experimental treatments in acute stroke. Ann Neurol. 2006;59:467-77.

69. de Boo J, Hendriksen C. Reduction strategies in animal research: a review of scientific approaches at the intra-experimental, supraexperimental and extra-experimental levels. Altern Lab Anim. 2005;33:369-77.

70. Dell RB, Holleran S, Ramakrishnan R. Sample size determination. ILAR J. 2002;43:207-13. 